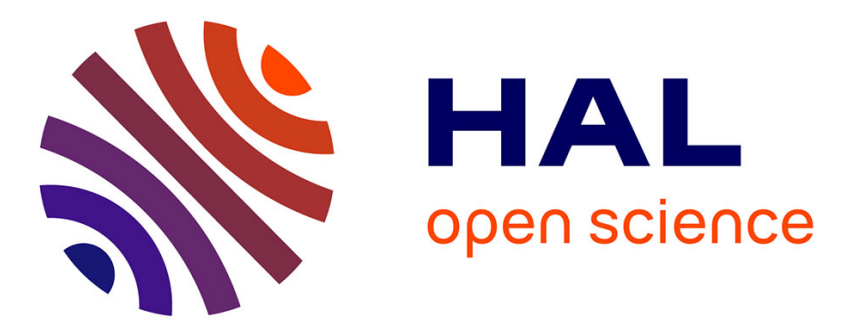

\title{
Domain adaptation for the extraction of complex urban patterns from multiresolution satellite images
}

\author{
Camille Kurtz, Anne Puissant, Nicolas Passat, Pierre Gançarski
}

\section{To cite this version:}

Camille Kurtz, Anne Puissant, Nicolas Passat, Pierre Gançarski. Domain adaptation for the extraction of complex urban patterns from multiresolution satellite images. International Geoscience and Remote Sensing Symposium (IGARSS), 2012, Munich, France. pp.1773-1776, 10.1109/IGARSS.2012.6351172 . hal-01695057

\section{HAL Id: hal-01695057 \\ https://hal.univ-reims.fr/hal-01695057}

Submitted on 15 Feb 2018

HAL is a multi-disciplinary open access archive for the deposit and dissemination of scientific research documents, whether they are published or not. The documents may come from teaching and research institutions in France or abroad, or from public or private research centers.
L'archive ouverte pluridisciplinaire HAL, est destinée au dépôt et à la diffusion de documents scientifiques de niveau recherche, publiés ou non, émanant des établissements d'enseignement et de recherche français ou étrangers, des laboratoires publics ou privés. 


\title{
DOMAIN ADAPTATION FOR THE EXTRACTION OF COMPLEX URBAN PATTERNS FROM MULTIRESOLUTION SATELLITE IMAGES
}

\author{
Camille Kurtz $^{\dagger}$, Student Member, IEEE, Anne Puissant ${ }^{\ddagger}$, Nicolas Passat ${ }^{\dagger}$ and Pierre Gançarski ${ }^{\dagger}$ \\ $\dagger$ LSIIT / University of Strasbourg, UMR CNRS 7005 - France \\ $\ddagger$ LIVE / University of Strasbourg, ERL CNRS 7230 - France
}

\begin{abstract}
The extraction of complex urban patterns from Very High Spatial Resolution (VHSR) images presents several challenges related to the complexity of the data. Based on the availability of images of a same scene at various resolutions (Medium to Very High Spatial resolutions), a hierarchical approach has been recently proposed to segment/classify objects of interest in a top-down fashion in order to determine patterns from VHSR images. To perform, this method requires the interactive definition of segmentation examples for each considered resolution image. In the context of large dataset processing, such interactive task becomes time consuming. To deal with this issue, we propose in this article, an extension of the domain adaptation paradigm enabling the transfer of the segmentation examples defined on a source dataset to automatically process a target one. Experiments performed on urban images provide satisfactory results which may be further used for operational needs.
\end{abstract}

Index Terms - Hierarchical segmentation, Clustering, Domain adaptation, Multiresolution satellite images, Urban analysis.

\section{INTRODUCTION}

$\mathbf{I}^{\mathrm{N}}$ $\mathrm{N}$ the field of Earth observation, a new generation of sensors of submetric resolution has led to the production of Very High Spatial Resolution (VHSR) optical images, and to an improved ability to analyze urban scenes. In such images, basic urban elements (e.g., houses, gardens, roads) are formed by different materials (e.g., red roofs, different kinds of asphalts or vegetations), while aggregate ones (e.g., urban districts, blocks) generally contain different kinds of basic elements with a specific spatial organization. For instance, a individual housing urban block is composed of a specific spatial organization of individual houses with gardens at a certain distance of a road. Thus, by opposition to lower resolution images, such complex patterns are not composed of homogeneous pixels but are often hierarchically organized (e.g., districts $\rightarrow$ blocks $\rightarrow$ basic elements). In the coming years, this phenomenon will be widely amplified thanks to the European PLEIADES program [1].

These properties, induced by VHSR images, lead to several new challenges. On one hand, the size and the complexity of the images make the visual analysis a time consuming and error prone task. On the other hand, new image analysis tools have to be developed since methods dedicated for lower resolutions, e.g., region-based ones (segmentation, classification) [2], are generally designed to extract segments based on radiometric homogeneous hypotheses.

In this context, and due to the actual importance to analyze VHSR images [3] in addition to lower spatial resolution ones,

The research leading to these results has received funding from the French Agence Nationale de la Recherche (Grant Agreement ANR-10COSI-012-03). it is then relevant to develop tools adapted to the extraction of complex patterns from such data. Moreover, the availability of data with a large range of spatial resolutions (from Medium Spatial Resolution (MSR, 30-5m) to VHSR ones) can enable the extraction of (potentially) hierarchical patterns [4], [5]. For instance, by analyzing first the image content at a coarse resolution and then gradually increasing this resolution, it is possible to detect complex patterns while avoiding the noise induced by the details [6].

Based on this consideration, a hierarchical approach has been proposed [7] to extract from multiresolution images, segments of interest from the lowest to the highest resolution data, and then finally determine complex patterns from VHSR images. This TopDown Hierarchical Approach (TDHA) has for purpose to use as much as possible the user's skills while making easier his interaction. To this end, at each resolution, an interactive segmentation of a sample region is required for each group of thematic ground areas of the image. Then, the user's behavior is learned and automatically reproduced in the remainder of the images.

Since the definition of these segmentation examples (on a specific dataset) is not a trivial task (i.e., time consuming task), and in view of the large amount of data that will be produced by the new incoming sensors, it could be relevant to maximize their potential by using them to deal with other datasets. To this end, several efforts have been conducted to design new approaches enabling such a learning transfer [8]. In particular, domain adaptation approaches enable to take advantage of the learned knowledge on a given source dataset in order to deduce a model appropriate for the processing of a similar target dataset.These approaches have been relevantly used for the classification of satellite images [9], [10].

In this article, we propose to extend the domain adaptation paradigm to enable the transfer of the segmentation examples required by the TDHA [7] segmentation approach to process one source dataset, in order to automatically segment a target dataset. Such an extension enables to assess the robustness of the TDHA approach by showing its capabilities on a domain adaptation task.

The article is organized as follows. Sec. II recalls the principles of the TDHA approach and describes the way to transfer the segmentation examples defined for one source dataset to a target one. Sec. III describes the domain adaptation experiments carried out with this methodology. Finally, Sec. IV concludes the article.

\section{METHODOLOGY}

\section{II-A. Hierarchical top-down approach}

The hybrid segmentation/classification approach proposed in [7] (TDHA) takes as input a multiresolution set of $n$ images $\left(\mathcal{I}_{1}, \ldots, \mathcal{I}_{n}\right)$ and provides as output $n$ classification maps computed in a top-down fashion. It performs $n$ successive steps (one per resolution image), each step being iteratively composed of: 


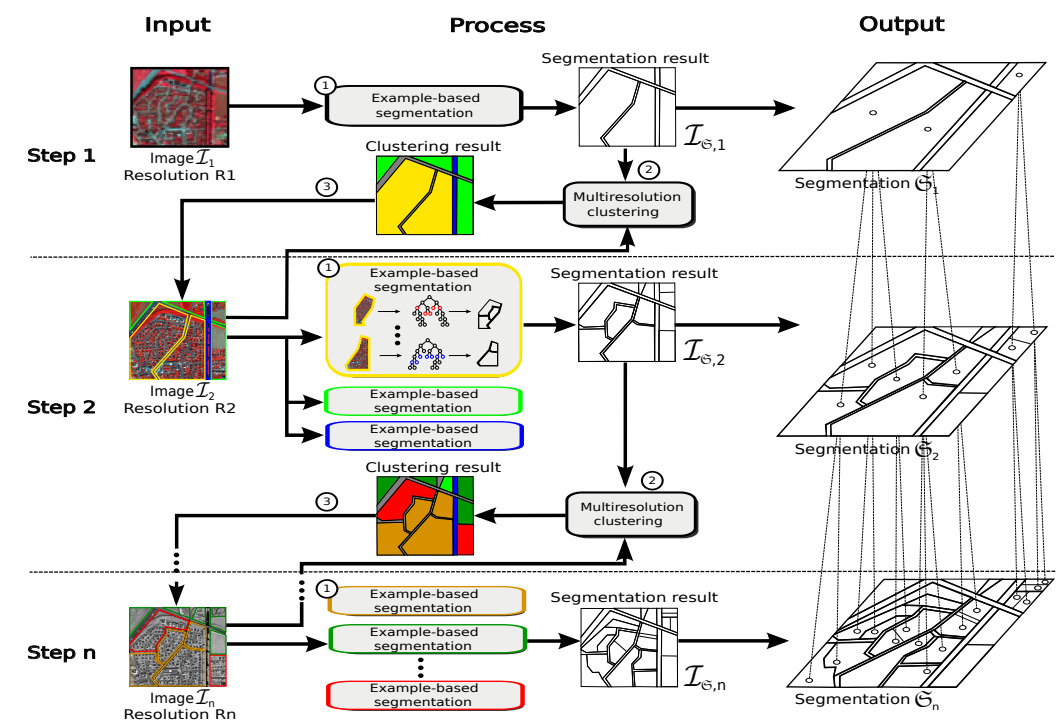

Fig. 1. Workflow overview of the top-down multiresolution hybrid approach proposed in [7].

- an example-based hierarchical segmentation approach (Fig. 1-1); - a multiresolution clustering approach (Fig. 1-(2).

At each resolution/step, the output of the process (namely a clustering map) is embedded into the next resolution image to be treated as input of the next step (Fig. 1-3).

Example-based hierarchical segmentation The segmentation of satellite images is not a trivial task. Indeed, the different objects of interest (and thematic ground areas) which are sensed by these images, cannot be segmented at the same scale. It is then difficult to correctly segment all these thematic ground areas by using only one segmentation result. To deal with this issue, the segmentation step is divided into $k$ different sub-steps (i.e., one sub-step per group of thematic ground areas). For each group of thematic ground areas of the image, it requires a segmentation example (of one representative area) interactively defined by the user (Fig. 1-(1)). The segmentation is performed by using a hierarchical strategy based on Binary Partition Trees (BPT) [11]. Thus, defining a segmentation example consists of cutting a BPT to produce a tree-cut example. Then, the user's behavior is learned using a learning strategy. Practically, the tree-cut example is summarized into a set of centroids (modelled by radiometric histograms) using a clustering algorithm. Finally, this tree-cut (segmentation) example is automatically reproduced in the similar thematic ground areas of the image by using a cutting strategy which processes all the remaining BPTs.

Multiresolution clustering The classification of the segments created by the previous step is performed by using a multiresolution clustering approach [12]. Instead of characterizing the segments extracted at the current resolution by using features computed on the current image, the segments are characterized by using their decompositions into the next resolution image (Fig. 1-(2)). To this end, for each segment, a class-based histogram is computed then modeling its composition in terms of radiometric clusters into the next resolution image. A classical clustering approach is then performed to create $c$ groups of segments sharing similar features. Once these groups have been created, the user can select and recognize them to match with potential thematic ground classes.

\section{II-B. Extension of the domain adaptation paradigm}

The classical domain adaptation approaches act by transferring the learned model (in general, a classifier) from a source to a target dataset for which a priori information is not (always) available. We propose hereafter an extension of such approaches enabling (in particular) the transfer of segmentation examples defined on a source dataset to automatically process a target one.

Transfer of the learned elements Once a source multiresolution set of images has been processed (i.e., segmented and classified), it becomes possible to transfer the learned segmentation examples and clusters to process a related target multiresolution set of images.

To perform, we transfer from the source dataset to the target one the following elements:

1) the $K$ tree-cut examples provided by the user (for each thematic ground area of each resolution image) during the example-based segmentation step

$$
K=k_{1}+\cdots+k_{n} \text { sets of histograms }
$$

These $K$ tree-cut examples can be directly used as input of the example-based segmentation approach to run the cutting strategy on the BPTs built for the different thematic ground areas of the images. This approach enables to automatically segment the $n$ images of the target dataset.

2) the $C$ centroids (of each resolution image) of the clusters learned during the multiresolution clustering step

$$
C=c_{1}+\cdots+c_{n} \text { centroids }
$$

These $C$ centroids can be used as input of the multiresolution clustering approach to classify the segments automatically created by the example-based segmentation approach.

Domain assumption As the learned segmentation examples and clusters are linked to specific thematic ground areas of the source dataset, we make the assumption that the target dataset is also composed of such ground areas. In the case of the thematic ground areas differ from the source dataset to the target one, the proposed domain adaptation strategy can be modified by adding or deleting a learned knowledge during the transfer step. 
Table I. Global evaluation results. F-measure and Kappa indexes.

\begin{tabular}{llcc} 
& & \multicolumn{2}{c}{ Evaluation indexes } \\
\hline Dataset & Level & $\overline{\mathcal{F}} \pm \sigma$ & $\mathcal{K} \pm \sigma$ \\
\hline DATASET-A & - Districts & $0.56 \pm 0.02$ & $0.71 \pm 0.02$ \\
& - Blocks & $0.69 \pm 0.01$ & $0.78 \pm 0.01$ \\
& - Basic elements & $0.72 \pm 0.03$ & $0.76 \pm 0.04$ \\
DATASET-B & - Districts & $0.67 \pm 0.03$ & $0.72 \pm 0.01$ \\
& - Blocks & $0.64 \pm 0.04$ & $0.75 \pm 0.05$ \\
& - Basic elements & $0.68 \pm 0.01$ & $0.69 \pm 0.01$ \\
\hline DATASET-B & - Districts & $0.65 \pm 0.01$ & $0.68 \pm 0.01$ \\
Domain adapt. & - Blocks & $0.61 \pm 0.02$ & $0.73 \pm 0.03$ \\
experiment & - Basic elements & $0.64 \pm 0.02$ & $0.66 \pm 0.01$ \\
\hline & & &
\end{tabular}

\section{EXPERIMENTS}

\section{III-A. Datasets and evaluation protocol}

We consider two datasets (denoted DATASET-A, -B) composed each of a SPOT-5 MSR $(9.6 \mathrm{~m})$ multispectral image and a couple of QUICKBIRD images composed by a HSR $(2.4 \mathrm{~m})$ multispectral image and a VHSR $(60 \mathrm{~cm})$ pan-sharpened one sensed over the urban area of Strasbourg (France). These images present two typical and similar suburban environments with (possible) water surfaces, forest areas, industrial areas, individual/collective housing blocks and agricultural zones. Fig. 2(a) (resp. Fig. 3(a)) presents the HSR image of the Dataset-a (resp. Dataset-B).

To assess the efficiency of the TDHA approach, experiments have been performed to extract from these datasets hierarchies of complex urban patterns (3 levels/scales: urban districts, blocks, basic elements). The results obtained have then been compared to certified ground-truth maps (one per considered scale) by using the F-measure $\overline{\mathcal{F}}$, and the Kappa $\mathcal{K}$ indexes. Fig. 2(b) (resp. Fig. 3(b)) presents the ground-truth map of the DATASET-A (resp. DATASETB) at the scale of the urban blocks.

To initialize the experiments, the DATASET-A has been processed using the TDHA approach. The example-based hierarchical segmentation step and the multiresolution clustering one have been iteratively run three times $(n=3)$ to extract the three considered scales. To perform, $K=16$ tree-cut examples have been provided by the expert $\left(k_{1}=1, k_{2}=8, k_{3}=7\right)$ and $C=26$ clusters have been extracted ( $\left.c_{1}=8, c_{2}=7, c_{3}=11\right)$. Fig. 2(c) presents the classification map obtained on the DATASET-A at the blocks scale.

\section{III-B. Domain adaptation experiment}

To validate the presented domain adaptation strategy (in the context of the TDHA approach), we have performed learning transfer experiments. The DATASET-A has been used as source dataset while the DATASET-B has been used as target dataset.

Practically, the $K=16$ tree-cut examples (provided by the user during the example-based segmentation step) and the $C=26$ centroids of the clusters (learned during the multiresolution clustering step) which are resulting from the processing of the DATASETA, have been transfered to automatically process the DATASET-B. However, as the thematic classes corresponding to water areas (blue class) and dense housing areas (red class) are not present in the DATASET-B, the segmentation examples and the learned clusters of the DATASET-A corresponding to these classes have not been transfered during the transfer step.

The results obtained are presented in Table I. From these results, one can note that the scores obtained on the DATASET-B are slightly

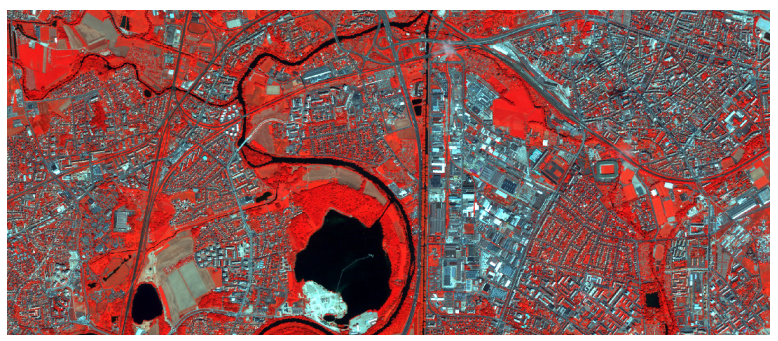

(a) HSR image (ㄷ DigitalGlobe Inc.).

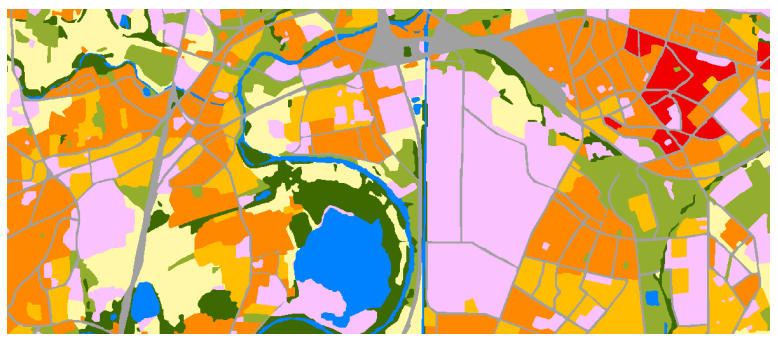

(b) Ground-truth map (urban blocks level, 9 classes).

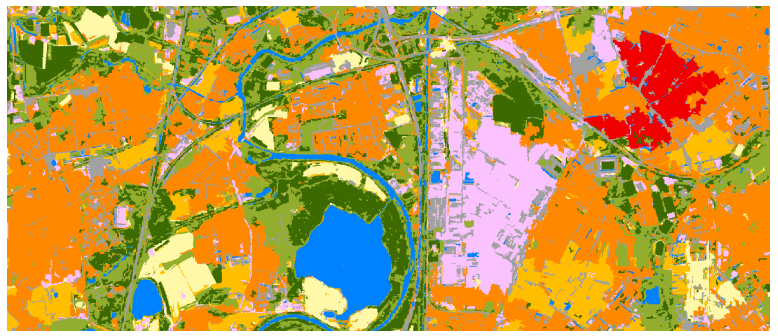

(c) Segmentation / classification result.

Fig. 2. Illustration of the DATASET-A and the associated result.

lower when the segmentation examples and the clusters learned are transferred from the DATASET-A (domain adaptation experiment) than when they are learned directly on the dataset. However, such results remain sufficient to further perform urban patterns detection. Furthermore, these experiments show that it is possible to maximize the potential of the user-defined segmentation examples (and thus to reduce the time spent by the user) to process several large datasets sensed over similar urban territories.

For a visual comparison purpose, the classification map obtained (at the blocks scale) is presented in Fig. 3(c) while the one resulting from the domain adaptation experiment is proposed in Fig. 3(d).

\section{PERSPECTIVES}

In this article we have presented an extension of the domain adaptation paradigm to enable the transfer of segmentation examples (required by the TDHA segmentation approach to process one source dataset), in order to automatically segment a target dataset.

Experiments performed on multiresolution satellite images were carried out to assess the robustness of the TDHA approach by showing its capabilities on a domain adaptation task. These experiments have provided satisfactory and accurate results which may be further used for operational needs.

This work opens up several perspectives. In particular, we plan to study more formally the robustness of the learning transfer step to deal with different kinds of urban cities. 


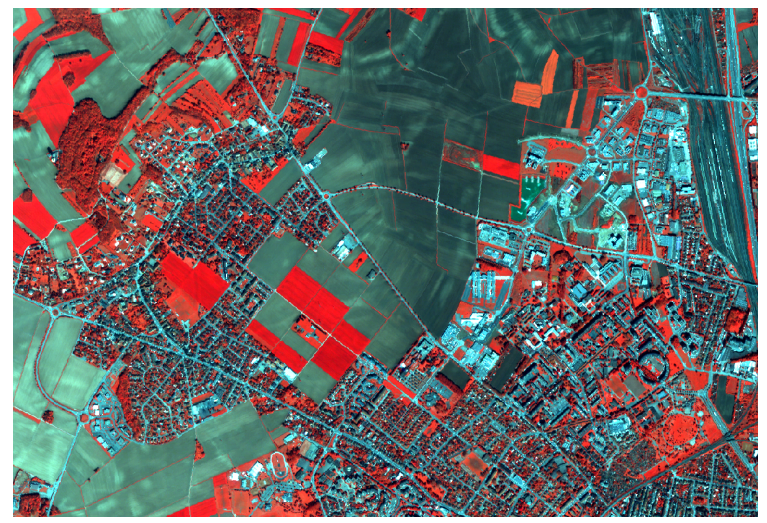

(a) HSR image (ㄷ DigitalGlobe Inc.).

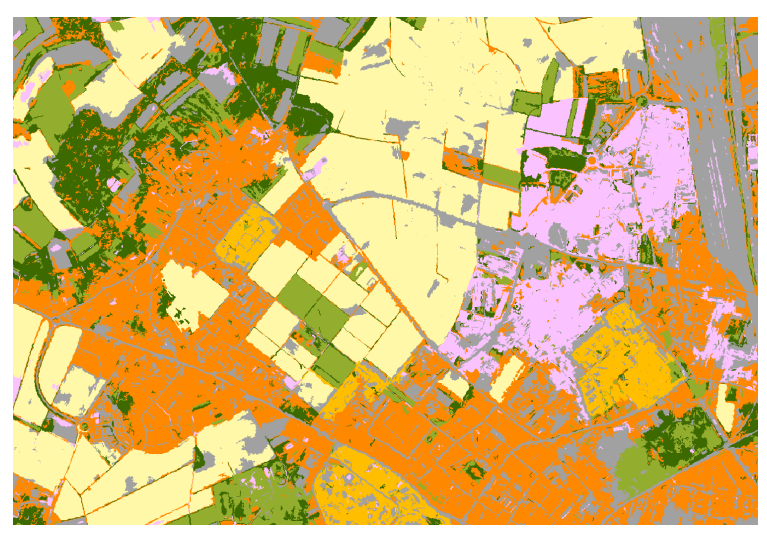

(c) Segmentation / classification result.

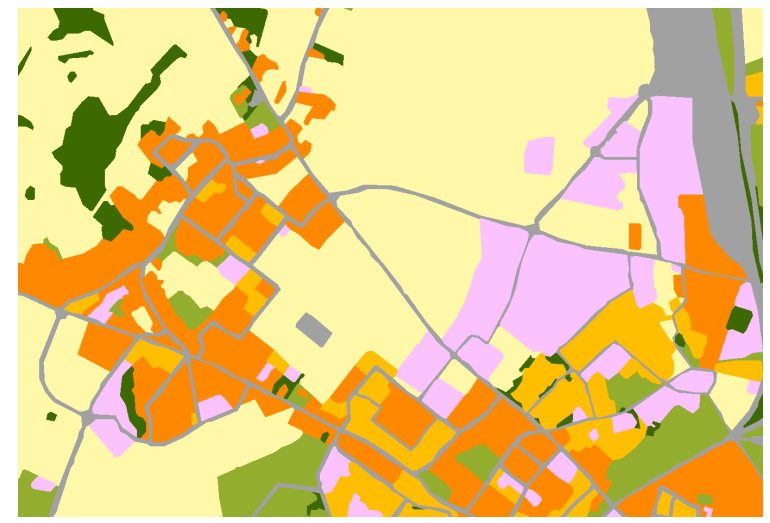

(b) Ground-truth map (urban blocks level, 7 classes).

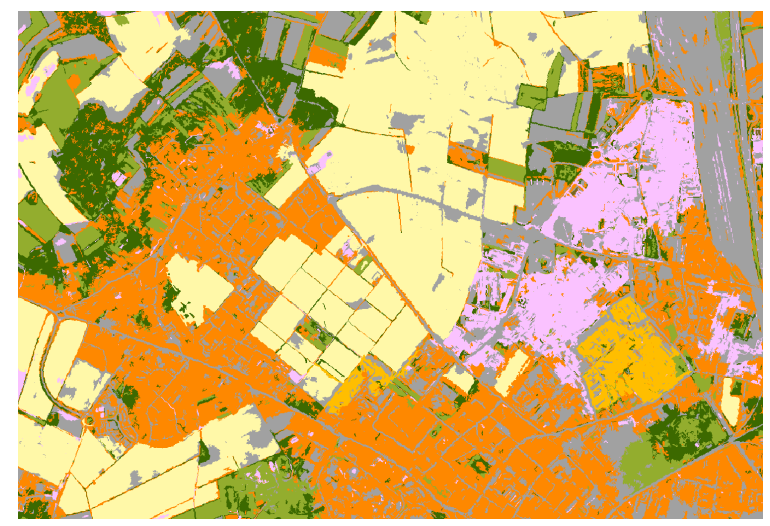

(d) Domain adaptation result (DATASET-A used as source dataset).

Fig. 3. Illustration of the DATASET-B and the associated results.

\section{REFERENCES}

[1] F. de Lussy, P. Kubik, D. Greslou, V. Pascal, P. Gigord, and J.P. Cantou, "PlEIADES-HR image system products and geometric accuracy," in Proceedings of the ISPRS Hannover Workshop on High-Resolution Earth Imaging for Geospatial Information, 2005, vol. 1, pp. 50-57.

[2] T. Blaschke, "Object based image analysis for remote sensing," ISPRS Journal of Photogrammetry and Remote Sensing, vol. 65, no. 1, pp. 2-16, 2010.

[3] A. Puissant and C. Weber, "The utility of Very High Spatial Resolution images to identify urban objects," Geocarto International, vol. 17, no. 1, pp. 33-44, 2002.

[4] H. G. Akcay and S. Aksoy, "Automatic detection of geospatial objects using multiple hierarchical segmentations," IEEE Transactions on Geoscience and Remote Sensing, vol. 46, no. 7, pp. 2097-2111, 2008.

[5] R. Gaetano, G. Scarpa, and G. Poggi, "Hierarchical texturebased segmentation of multiresolution remote-sensing images," IEEE Transactions on Geoscience and Remote Sensing, vol. 47, no. 7, pp. 2129-2141, 2009.

[6] W. Sun, V. Heidt, P. Gong, and G. Xu, "Information fusion for rural land-use classification with high-resolution satellite imagery," IEEE Transactions on Geoscience and Remote Sensing, vol. 41, no. 4, pp. 883-890, 2003.
[7] C. Kurtz, N. Passat, P. Gançarski, and A. Puissant, "Extraction of complex patterns from multiresolution remote sensing images: A hierarchical top-down methodology," Pattern Recognition, vol. 45, no. 2, pp. 685-706, 2012.

[8] H. Daumé III and D. Marcu, "Domain adaptation for statistical classifiers," Journal of Artificial Intelligence Research, vol. 26, no. 1, pp. 101-126, 2006.

[9] L. Bruzzone, D.F. Prieto, and S.B. Serpico, "A neuralstatistical approach to multitemporal and multisource remotesensing image classification," IEEE Transactions on Geoscience and Remote Sensing, vol. 37, no. 3, pp. 1350-1359, 1999.

[10] K. Bahirat, F. Bovolo, L. Bruzzone, and S. Chaudhuri, "A novel domain adaptation Bayesian classifier for updating land-cover maps with class differences in source and target domains," IEEE Transactions on Geoscience and Remote Sensing, vol. (In Press), 2012.

[11] P. Salembier and L. Garrido, "Binary partition tree as an efficient representation for image processing, segmentation, and information retrieval," IEEE Transactions on Image Processing, vol. 9, no. 4, pp. 561-576, 2000.

[12] C. Kurtz, N. Passat, P. Gançarski, and A. Puissant, "Multiresolution region-based clustering for urban analysis," International Journal of Remote Sensing, vol. 31, no. 22, pp. 5941-5973, 2010. 